\title{
X-ray line profiles from structured stellar winds
}

\author{
Lidia M. Oskinova, Achim Feldmeier, and Wolf-Rainer Hamann \\ Lehrstuhl Astrophysik, Institut für Physik, Universität Potsdam, \\ Am Neuen Palais 10, D-14469 Potsdam, BRD
}

\begin{abstract}
Absorbing material compressed in a number of thin shells is effectively less opaque for $\mathrm{X}$-rays than smoothly distributed gas. The calculated $\mathrm{X}$-ray emission-line profiles are red-shifted if the emission arises from the starward side of the shells.
\end{abstract}

There is a widespread view, that instabilities in the line-driving mechanism which accounts for the stellar wind should generate strong shocks. Numerical simulations by Feldmeier et al. (1997) demonstrate that the X-rays occur over a narrow range of radii close to the star, when fast moving blobs ram into the starward side of larger shells. Then the X-rays emitted away from the star, which form the blue part of the observed line profile, immediately have to pass through the dense material of their natal shell, suffering therefore strong absorption. Xrays emitted by material moving in a negative radial direction, which form the red part of the observed line, do not have to pass through the material of the native shell. Some X-rays have a chance to emerge from the wind without intersecting any shell, reducing the wind opacity.

We calculate line profiles by formal integration of the radiative transfer equation. All material of the wind in our model is fragmented in a number of shells, but X-rays originate only over a range of radii close to the star. Two cases were considered. In Case I, X-rays are produced only at the starward side of the shells. In Case II, the X-ray emitting material is distributed randomly over the same range of radii as in Case I, but not coupled with the shells. The key difference between those two cases is, that X-ray photons emitted by material moving towards the observer and forming blue part of an emission line are always suffering absorption in Case I, but have some probability to escape unabsorped in Case II.

A 2D cut through a spherical wind $(r, \theta)$ is divided into $N_{\theta}$ radial cones. Each cone contains material compressed in $N_{\mathrm{r}}$ radial shells. The space between subsequent shells is void, but the total radial optical depth is that of a homogeneous wind. The shells are located at random distances bigger than initial $R_{\text {inner }}$. Shells expand radially with constant velocity $v_{\infty}$. The vicinity of the star till $R_{\text {inner }}$ is filled with homogeneous material moving with velocity $v \propto r$. The optical depth of such a material is $\tau \propto(r \operatorname{tg} \theta \sin \theta)^{-1}$. A sketch of the model is shown in the left panel of the Figure 1. The total number of shells used in the simulations is of the order of $N_{\theta} \times N_{\mathrm{r}} \approx 10^{5}$. To compensate for the 2D approach and average the result over azimuth, the simulations of the line profile are performed $N_{\theta}$ times and averaged. 

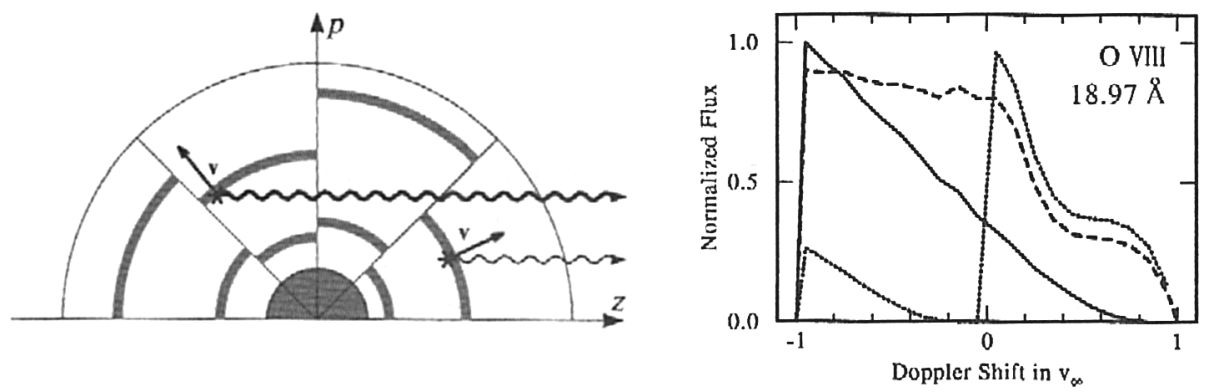

Figure 1. Left: sketch of the blob-shell collision model. Optically thick material is fragmented in radial shells expanding with constant velocity $v$. The vicinity of the star inside the radius $R_{\text {inner }}$, where the fragmentation occurs, is filled with homogeneous material moving with $v \propto r$ (grey circle). The thick black line indicates the path along which photons move towards the observer without being intersected by a shell. The thin black line indicates the path of photons which suffered absorption. Right: line profiles of $\mathrm{O}$ VIII for the parameters of $\zeta$ Pup (Puls et al. 1996). The full-drown line represents the case when the wind is homogeneous. The dashed line represents Case I. The opacity is reduced only for the photons originated in the red part of the line, but those in the blue part are severely depleted by absorption in the natal shell. The dotted line represents Case II. The opacity is reduced and the line is more centered, compared to the homogeneous case. This case resembles most the observed line profiles (e.g., Cassinelli et al. 2001). The shoulder in the red part of the lines is due to the absorption by the homogeneous inner wind.

Our simulations reveal that:

(i) the structured absorbing material is effectively less opaque than the homogeneously distributed one (see also Feldmeier et al. in these Proceedings). Averaged over angle, the probability for an X-ray photon to pass throughout the wind without intersecting a shell is $\beta=2 /\left(N_{\mathrm{r}}+2\right)$, if hot plasma fills the whole volume of the wind, and $\beta=\exp \left(-N_{\mathrm{r}}\right)$, when the emission originates only from close to the star. The effects caused by the fragmentation of the optically thick general wind are significant when the number of fragments in radial direction is small;

(ii) when photons from the front hemisphere always have to cross their natal shell (Case I), more photons emerge in the red part of the line than in the blue part, making the line skewed and red-shifted. Since the absorption is strongest in the line center, the profiles show a central depression (Figure 1); and

(iii) when X-ray emitting material is distributed randomly (Case II), the line profile is skewed and blue-shifted. The degree of skewness depends on the distribution of the opaque material. The opacity is reduced and the line is more centered for a fractured wind as compared to the case of the homogeneous opaque material (Figure 1).

\section{References}

Cassinelli, J.P., Miller, N.A., Waldron, W.L., et al. 2001, ApJ 554, 55

Feldmeier, A., Puls, J., Pauldrach, A.W.A. 1997, A\&A 322, 878

Puls, C.A., Kudritzki, R.-P., Herrero, A., et al. 1996, A\&A 305, 171 